\title{
Spontaneous emission in confined space according to stochastic electrodynamics
}

\author{
H. M. França, ${ }^{*}$ T. W. Marshall, ${ }^{\dagger}$ and E. Santos \\ Departamento de Física Moderna, Universidad de Cantabria, 39005 Santander, Spain
}

(Received 13 August 1990; revised manuscript received 19 August 1991)

\begin{abstract}
Modeling an atomic excited state as a simple charged dipole oscillator immersed in a random (zeropoint) radiation, we discuss the effects of two metallic plates on the properties of a microscopic system. The spectral distribution of the zero-point electromagnetic field, characteristic of stochastic electrodynamics, and the rate of emission of the oscillator are modified by the boundaries of the cavity. As a result, the lifetime of the oscillator excited states are different from the free-space values. A comparison with recent experimental results [W. Jhe et al., Phys. Rev. Lett. 58, 666 (1987)] exhibiting suppression of spontaneous decay of excited Cs atoms shows a good agreement with our simplified model calculation.
\end{abstract}

PACS number(s): 32.70.Fw, 03.65.Sq

\section{INTRODUCTION}

The role of zero-point electromagnetic radiation in many physical phenomena is becoming clearer since its first appearance in physics with Planck's second blackbody theory [1]. A few years after Planck's discovery of these vacuum zero-temperature electromagnetic fluctuations, Nernst [2] proposed that zero-point radiation might be responsible for the stability of atomic systems. With the development of quantum mechanics in the 1920 s, zero-point radiation reappeared as a straightforward consequence of field quantization. However, it lost the status of a real field to become a "virtual" field, that is, it could not be observed directly. Nevertheless, important effects of the zero-point radiation on physical systems were discovered [3]: radiative corrections to the atomic energy levels, anomalous magnetic moment of the electron, the Casimir effect [4] (forces between atoms and macroscopic objects), etc. Later on, the zero-point fields regained more attention with the works by Welton [5] and Sokolov and Tumanov [6], where these quantum electromagnetic fluctuations were considered as the source of the microscopic fluctuations presented by the electron inside an atom.

The experimental confirmation of these theoretical predictions, like, for instance, the measurements of the radiative correction to the $\mathrm{H}$-atom fine structure (Lamb shift [7]) and the Casimir attraction between conducting plates observed by Sparnaay [8], were considered as spectacular successes of quantum electrodynamics.

More recently, with the use of lasers, it has been possible to study environmental changes of the vacuum fluctuations, for instance, using highly excited states of atoms which have large polarizabilities and therefore are strongly coupled with the electromagnetic field. If the atom is inside a cavity, its radiative properties are modified because the electromagnetic field surrounding the atom can be drastically altered by the presence of the cavity walls. These effects have been observed experimentally and have generated a new field called "cavity quantum electrodynamics" [9].

A very simple system in which one can understand the important role of zero-point electromagnetic fields is the charged harmonic oscillator. Moreover, by Ehrenfest's theorem, the expectation values of any quantummechanical quantity that has linear Heisenberg equations of motion will be identical to the corresponding classical one and, therefore, quite easy to interpret. Within the realm of nonrelativistic quantum electrodynamics (QED) the Heisenberg equation of motion for this system takes the form $[6,10]$

$$
\ddot{x}=-\omega_{0}^{2} x+\frac{2}{3} \frac{e^{2}}{m c^{3}} \dddot{x}+\frac{e}{m} E_{x}(t),
$$

where $\omega_{0}$ is the natural frequency of the oscillator, $E_{x}(t) \equiv-(1 / c) \partial A_{x} / \partial t$ is the quantized electric field, and $2 e \dddot{x} / 3 c^{3}$ is the radiation reaction or self-field. These electromagnetic fields represent, respectively, the fluctuation and the dissipation that govern the dynamics of such a microscopic system because even at zero temperature there are vacuum fluctuations associated with the field operator $E_{x}(t)$. Therefore it is possible to consider these fluctuations as the source of quantum fluctuations on the position $x$ of the oscillating charge. In fact, it is not difficult to derive the quantum commutation relation between the position and momentum of the oscillator as is well known [10-12]. From the stationary solution of (1.1) one can show that the commutation relation between $x$ and the canonical momentum $p_{c}=m \dot{x}$ $+(e / c) A_{x}-2 e^{2} \ddot{x} / 3 c^{3}$, namely $[6,10]$

$$
\left[x, p_{c}\right]=\frac{i \hbar 2 e^{2}}{\pi 3 m c^{3}} \int_{0}^{\infty} d \omega \frac{\omega^{4}}{\left(\omega^{2}-\omega_{0}^{2}\right)^{2}+\left[\frac{2}{3} \frac{e^{2} \omega^{3}}{m c^{3}}\right)^{2}}=i \hbar
$$

follows from the commutation relations associated to the zero-point electromagnetic fields (observe that this is so only if the dissipative force is precisely $\left.2 e^{2} \dddot{x} / 3 c^{3}\right)$. It is also easy to show that the ground-state energy of the three-dimensional oscillator is $3 \hbar \omega_{0} / 2$ (see Ref. [10] for instance). This happens because, in equilibrium, the emitted radiation is balanced by the energy absorbed from 
the quantum zero-point electromagnetic fields. In other words, the rate of exchange of energy between the charge and the total radiation field $E=E_{x}+2 e \dddot{x} / 3 c^{3}$ is such that $[13,14]$

$$
\begin{aligned}
\frac{e}{2}\langle n|(\dot{x} E+E \dot{x})| n\rangle & =-\frac{4}{3} \frac{e^{2}}{c^{3}} \sum_{n^{\prime}(<n)}\left\langle n|\ddot{x}| n^{\prime}\right\rangle\left\langle n^{\prime}|\ddot{x}| n\right\rangle \\
& =-\frac{2}{3} \frac{e^{2} \omega_{0}^{2}}{m c^{3}}\left(\varepsilon_{n}-\varepsilon_{0}\right),
\end{aligned}
$$

where $|n\rangle$ denotes an arbitrary state [energy $\left.\varepsilon_{n}=\hbar \omega_{0}\left(\frac{1}{2}+n\right)\right]$ of the oscillator in free-space vacuum. This interesting result means that [13] (1) the charge in the vacuum can only lose energy by cascading downwards to lower energy levels; (2) the ground state cannot be stable in the absence of the vacuum free-space fluctuations which exactly balance the energy loss due to self reaction. One can easily obtain from (1.3) the value of the Einstein $A$ coefficient of "spontaneous" emission and the lifetime of the excited state in the free-space vacuum. It was also shown in Refs. [13] and [14] that conclusions (1) and (2) are valid for real atoms which are much more complicated than simple oscillators. As far as we know this was the first confirmation, within the realm of QED, of the proposal made by Nernst [2] more than 70 years ago. However, within Nernst's original scheme, the vacuum zero-point fluctuations were associated with classical electromagnetic fields.

If the radiating system is inside a cavity (near conducting plates for instance) it is expected that some of the properties of this microscopic system will be modified [9]. We address ourselves to discuss this problem here because of its fundamental relevance to our understanding of the dynamics of the microscopic world. Due to recent advances in experimental techniques, physicists are learning how to modify the behavior of atoms [9]. The zeropoint electromagnetic field has a fundamental role in these attempts, as we shall see.

In order to discuss these points we prefer to work within the realm of stochastic electrodynamics [15-22] (SED) instead of using the standard QED formalism. The reason for this choice is that within SED the zeropoint radiation is a real classical (stochastic) electromagnetic field, whereas in QED these vacuum fields are very often considered "virtual," a not-well-defined concept in our opinion [23].

Stochastic electrodynamics is just classical electrodynamics with the hypothesis of a random background radiation in the whole space (zero-point radiation). The reasoning which leads to this idea can be summarized as follows. In space there are systems of charged particles (atoms or molecules) that move according to the classical laws. These atoms will be continuously radiating and therefore some amount of radiation will be always present in space. The radiation will act on the atoms and these will arrive at a state of dynamical equilibrium such that the rate of emission equals the rate of absorption. This may explain, in a simple way, the stability of atoms without departing from classical theories. Once the existence of some amount of radiation in space is assumed, its spectral density is fixed by very general principles. In fact, the only spectrum which is Lorentz invariant has a density $\rho_{0}(\omega)$ such that

$$
\rho_{0}(\omega)=\text { const } \times \omega^{3} \equiv \hbar \omega^{3} / 2 \pi^{2} c^{3} .
$$

This background radiation is such that we have a mean energy of $\hbar \omega / 2$ associated with each normal mode of the electromagnetic fields. If we postulate the existence of classical electromagnetic (fluctuating) fields, persisting even at zero temperature, classical electrodynamics is provided with a new boundary condition. In this way some of the quantum behavior of the microscopic matter can be predicted entirely on classical grounds [2]. Some examples may be found in the reviews by Boyer [18,21], Milonni [19], Claverie and Diner [20], and de la Peña [22] where the microscopic properties of the harmonic oscillator, the blackbody radiation, the diamagnetic behavior of free and harmonically bound charges, the Casimir forces between macroscopic objects, and other phenomena are discussed. More recently it has been shown that the paramagnetic behavior of a rigid magnetic dipole [24], the specific heat of solids [25], and also other fundamental electromagnetic processes [26] may be understood classically.

With the purpose of a clear presentation of another example of the above-mentioned ideas, we propose to discuss in this paper a simple model which describes the emission of radiation by an excited atomic state, when the atom is between two mirrors. In our simplified description we shall assume that the rate of energy emitted during the transition is the same as the rate of emission by an oscillator. With this goal in mind our paper is organized as follows. For the reader's convenience we present in Sec. II a summary of previous results as far as the behavior of an oscillator between conducting plates is concerned. We want to draw the reader's attention to the oscillator properties which are modified and also to those which are not modified by the changes in the zero-point modes of the electromagnetic field due to the presence of the metallic plates. In Sec. III we discuss how the spontaneous emission of the oscillator excited states is modified in confined space. We also present, in Sec. III, a comparison between our model calculation and some recent experimental results. In Sec. IV we address ourselves to a qualitative discussion of atomic stability $[13,18]$. Our goal is to stress the role of zero-point radiation as a source of energy which maintains the groundstate stability (the emitted radiation is compensated by the radiation absorbed from zero-point electromagnetic fields), which is the essential idea of SED. Finally we present our conclusions in Sec. IV.

\section{DIPOLE BETWEEN CONDUCTING PLATES}

As a simple example that can illustrate how the environment can change the behavior of a microscope system, we shall consider a dipole oscillator between two large conducting plates separated by the distance $a$. Since, very often, one can consider an atomic excited state (with large polarizability) as an oscillating electric dipole emitting radiation [27], the theoretical implications of this example can be checked experimentally, as 
we shall see.

The phenomenon that we are going to study in Sec. III is the spontaneous emission by an excited state. In quantum language, the emission can be suppressed if the emitted photon (wavelength $\lambda$ ) corresponds to a mode which cannot propagate between the plates $(\lambda>2 a$ for photon polarization parallel to the mirror plates). Since the criteria for the propagation are derived from classical Maxwell theory and since QED makes a distinction between real photons (emitted by the atoms) and the virtual photons from the quantum vacuum, we prefer to work within the realm of classical stochastic electrodynamics as we have mentioned in the Introduction. Within SED all modes of the electromagnetic fields are treated on the same footing, that is, they correspond to real modes that interact with the atomic dipole.

This problem was studied by Marshall [16], who obtained the main results we are going to use in Sec. III. The same questions were discussed later by Milonni and Knight [28] using the quantum formalism. More recently Cetto and de la Peña [29] considered again the problem within the realm of SED. In all these papers $[16,28,29]$ the authors reach essentially the same conclusions. For the reader's convenience we are going to summarize here the results which we shall use in Sec. III.

If we have an oscillating dipole (frequency $\omega$ ) that is between two parallel perfectly conducting plates, the emission rate is different from the free-space emission because the fields reflected by the mirror plates interfere with the emitted wave. If the interference is constructive we have enhanced emission or, alternatively, one can obtain an inhibited emission in the case of destructive interference. The phenomenon is typically undulatory in character.

Therefore if we have an oscillating electric dipole located at the point $(0,0, b)$ in between two mirrors separated by the distance $a(a>b)$, the emitted power must be calculated taking into account the whole set of image dipoles (we are assuming that the plates are perpendicular to the $z$ direction and are located at $z=0$ and $a$ ). Since the image dipoles are located at the points $z=b \pm 2 a, b \pm 4 a, \ldots$ and at $z=-b,-b \pm 2 a,-b \pm 4 a$ ,..., one can calculate the total electric field at the position $(0,0, b)$ of the real dipole.

The retarded fields generated by an oscillating dipole are well known [30]. However, we only need to take into account those fields which contribute to the emission and absorption of radiation by the real dipole [16]. Of course there are terms that give rise to a van der Waals force between the real dipole and conducting plates [32]. This force will contribute to displace the dipole as a whole and will not be considered here.

The above discussions shows that the spontaneous emission by the oscillator excited states is modified by the conducting plates. The equations of motion, which are isotropic in free space, are changed to [16]

$$
\begin{aligned}
\ddot{x}+\gamma_{x} \dot{x}+\omega^{2} x & =\frac{e}{m} E_{x}, \\
\ddot{y}+\gamma_{y} \dot{y}+\omega^{2} y & =\frac{e}{m} E_{y}, \\
\ddot{z}+\gamma_{z} \dot{z}+\omega^{2} z & =\frac{e}{m} E_{z},
\end{aligned}
$$

if the oscillator is between two conducting plates. In this case we must take $\gamma_{x}=\gamma_{y}=\gamma_{\|}$and $\gamma_{z}=\gamma_{\perp}$. Here $E_{x}, E_{y}$, and $E_{z}$ are the components of the electric field associated with the zero-point radiation. These fluctuating fields are also modified by the metallic boundaries [16,29].

In free space the damping constants are such that $\gamma_{x}=\gamma_{y}=\gamma_{z}=\gamma_{\mathrm{FS}}$ and

$$
\gamma_{\mathrm{FS}}=\frac{2}{3} \frac{e^{2}}{m c^{3}} \omega^{2}
$$

which is the well-known expression for the damping constant [31]. However, for an oscillator at the point $(0,0, b)$ between two metallic plates (see Fig. 1) we have

$$
\frac{\gamma_{\perp}}{\gamma_{\mathrm{FS}}}=\frac{3 \pi c}{\omega a}\left\{\frac{1}{2}+\sum_{s=1}^{[\omega a / \pi c]}\left[1-\left(\frac{\pi c s}{\omega a}\right)^{2}\right] \cos ^{2}\left(\frac{\pi b s}{a}\right)\right\}
$$

and

$$
\frac{\gamma_{\|}}{\gamma_{\mathrm{FS}}}=\frac{3 \pi c}{2 \omega a} \sum_{s=0}^{[\omega a / \pi c]}\left[1+\left(\frac{\pi c s}{\omega a}\right)^{2}\right] \sin ^{2}\left(\frac{\pi b s}{a}\right),
$$

where $[\omega a / \pi c]$ is the integral part of the ratio $\omega a / \pi c$.

As far as we know these expressions were obtained for the first time by Marshall [16]. More recently Cetto and de la Peña [29] rederived the expressions without using the image method. This method, however, was used by Milonni and Knight [28] within the realm of the quantum theory. The quantum result can be obtained from (2.3) and (2.4) by replacing $\gamma_{\text {FS }}$ by the Einstein $A$ coefficient of spontaneous emission. We can understood this (within the realm of SED) if we recall a previous result by França and Marshall [33]. By studying transitions between oscillator states with energies $\hbar \omega\left(n+\frac{1}{2}\right)$ these authors obtained for the Einstein $A$ coefficient (corresponding to a downward transition) the following result valid in free space:

$$
A(n \rightarrow n-1)=\frac{1}{3} \frac{e^{2} \omega^{2}}{m c^{3}}\left(1+\frac{2 \pi^{2} c^{3}}{\hbar \omega^{3}} \rho_{0}(\omega)\right) n=\gamma_{\mathrm{FS}} n
$$

Here one can easily see that the Einstein coefficient of spontaneous emission has contributions from the radiation reaction field and also from zero-point electromagnetic fields [see also (1.3) and (1.4)].

We have already mentioned that the fluctuating fields of the zero-point electromagnetic radiation are also modified by the metallic boundaries. Marshall [16] was able to calculate the spectral density of modes by requiring that each normal mode has an average energy $1 / 2 \hbar \omega$. This is the usual hypothesis in SED, the same as in QED (in free space this corresponds to the requirement of a Lorentz invariant spectral distribution for the zero-point electromagnetic radiation). Using the modified spectral distribution and also (2.3) and (2.4), Marshall was able to show that the statistical properties associated with the ground state of the oscillator are preserved, that is, are the same as those valid for free space. In other words the ground-state average energy is precisely $3 \hbar \omega / 2$ and the 
probability distribution is a Gaussian with variances

$$
\left\langle x^{2}\right\rangle=\left\langle y^{2}\right\rangle=\left\langle z^{2}\right\rangle=\frac{\hbar}{2 m \omega} .
$$

Here the frequency $\omega$ must be greater than $\pi c / a$ if we have perfectly conducting plates [see (2.4)]. Also the oscillator phase-space distribution is the same as in free space. If the oscillator is forced by an additional deterministic external force (with arbitrary time dependence) it is easy to show that coherent states are generated in the same way as we have seen before $[33,34]$.

There are also other properties of the oscillator which are affected by the environmental modifications of the electromagnetic vacuum. They are mass corrections and Lamb shift corrections to the energy. However, they are very small as one can see from the more recent works by Cetto and de la Peña [29]. Hence the environmental effects due to the presence of the plates show up mainly through the modifications displayed by $\gamma_{\|}$and $\gamma_{\perp}$. This affects the lifetime of excited states and can be observed experimentally, as we shall see in Sec. III.

\section{SUPPRESSION OF SPONTANEOUS DECAY IN CONFINED SPACE}

Let us consider an atom inside a metallic cavity. The structure of the spectral distribution of zero-point radiation is dramatically altered in the wavelengths comparable to the dimensions of the cavity [16,29]. In the case of two perfect mirrors, for instance, $\gamma_{\|}$will be zero for wavelengths larger than $2 a$ as one can see from (2.4).

Very recently, interesting experiments were carried out using the parallel-mirror geometry we have discussed in the preceding section. One experiment demonstrated the inhibition of spontaneous emission from Rydberg states of cesium atoms [35]. A beam was passed through a tunnel between two mirrors separated by a distance $a$ such that $a<\lambda / 2$ where $\lambda$ is the wavelength of the emitted radiation. The atoms surviving in the initial quantum state were detected at tunnel exit by ionizing them in a small electric field. The lifetime obtained is at least 20 times larger than it is in free space.

A similar experiment was performed by Jhe et al. [36]. In this experiment the inhibited transition was $5 D_{5 / 2} \rightarrow 6 P_{3 / 2}$ at a wavelength of $3.49 \mu \mathrm{m}$. The excited atoms propagate through the tunnel (between two metallic mirror separated by a $1.1-\mu \mathrm{m}$ gap) for about 13 natural lifetimes without appreciable decay. The experimenters applied a small magnetic field $(2.4 \mathrm{G})$ in order to demonstrate the anisotropy of spontaneous emission between mirrors. The magnetic dipole $\mu=(e / 2 c) \mathbf{r} \times \dot{\mathbf{r}}$ associated with the excited state will precess around the applied magnetic field $\mathbf{B}$. The electric dipole $\mathbf{p}=e \mathbf{r}$, which is always perpendicular to $\mu$, will change its orientation in space and the spontaneous-emission rate will be different for different magnetic-field orientations. Modeling an atomic excited state as a simple dipole oscillator immersed in the zero-point radiation, we want to see the effects of two metallic plates on the properties of this microscopic system.

Let us assume that the magnetic field $\mathbf{B}$ is oriented in such a way that it makes an angle $\theta$ with the $z$ direction, which we have taken as perpendicular to the mirrors. The magnetic moment $\boldsymbol{\mu}$ will precess around the $\mathbf{B}$ direction with the Larmor frequency $\omega_{B}=e B / 2 m c$. It is easy to show that the orientation of the vector $\mu$ will change in time according to

$$
\begin{aligned}
& \mu_{x} / u=\cos \theta \sin \varphi \cos \left(\omega_{B} t\right)+\sin \theta \cos \varphi, \\
& \mu_{y} / \mu=\sin \varphi \sin \left(\omega_{B} t\right), \\
& \mu_{z} / \mu=-\sin \theta \sin \varphi \cos \left(\omega_{B} t\right)+\cos \theta \cos \varphi,
\end{aligned}
$$

where $\mu \equiv|\boldsymbol{\mu}|$ is assumed to be constant and the angle $\varphi$ is defined as the initial orientation of $\mu$ with respect to the direction of the magnetic field $\mathbf{B}$. This is illustrated in Fig. 1.

We will also assume for simplicity that the electron motion in the excited state corresponds to a circular orbit with a frequency $\omega$. Therefore the electric dipole p, which is always perpendicular to $\mu$, will have an orientation in space that is easy to relate to the orientation of the vector $\boldsymbol{\mu}$. If the instantaneous orientation of $\boldsymbol{\mu}$ has a polar angle $\psi(t)$ and an azimuthal angle $\phi(t)$ one can show that

$$
\begin{aligned}
& p_{x} / p=\cos \psi \cos \phi \sin (\omega t)+\sin \phi \cos (\omega t), \\
& p_{y} / p=-\cos \psi \sin \phi \sin (\omega t)+\cos \phi \cos (\omega t), \\
& p_{z} / p=\sin \psi \sin (\omega t),
\end{aligned}
$$

where $p=|\mathbf{p}|$ is a constant for circular orbits. Within this model one can consider that the excited state emits radiation like a dipole oscillator with a frequency $\omega$. Therefore the emitted radiation will have a wavelength $\lambda=2 \pi c / \omega$.

In order to apply the results of Sec. II we must obtain the effective damping constant $\left(\Gamma_{\text {eff }}\right)$ associated with the dipole motion described by (3.1) and (3.2). Since one can define the effective damping constant as the ratio between the average emitted power and average oscillator energy we get

$$
p^{2} \Gamma_{\mathrm{eff}}=\gamma_{x} \bar{p}_{x}^{2}+\gamma_{y} \bar{p}_{y}^{2}+\gamma_{z} \bar{p}_{z}^{2},
$$

where the bar denotes times average. The damping constants $\gamma_{i}$ were obtained previously, that is, $\gamma_{x}=\gamma_{y}=\gamma_{\|}$

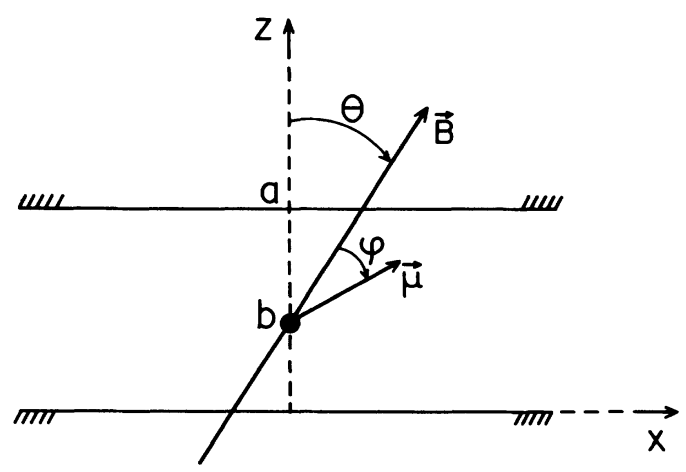

FIG. 1. Initial orientation of the magnetic moment $\mu$ with respect to the excited state. 
and $\gamma_{z}=\gamma_{1}$. Of course, $\Gamma_{\text {eff }}$, besides depending on the magnetic-field orientation $\theta$, depends on the position occupied by the atom and on the distance between the mirrors, as one can see from (2.3) and (2.4).

Using (3.2) one can express $\Gamma_{\text {eff }}$ as

$$
2 \Gamma_{\text {eff }}(\theta, \varphi)=\gamma_{\|}\left(1+\overline{\cos ^{2} \psi}\right)+\gamma_{\perp}\left(1-\overline{\cos ^{2} \psi}\right),
$$

and since $\cos \psi=\mu_{z}(t) / \mu$ we get

$$
\overline{\cos ^{2} \psi}=\cos ^{2} \theta \cos ^{2} \varphi+\frac{1}{2} \sin ^{2} \theta \sin ^{2} \varphi,
$$

where the angle $\varphi$ defines the initial orientation of the excited-state magnetic dipole $\mu$ with respect to the magnetic field B (see Fig. 1). Since, in the atomic beam which enters the tunnel between the mirrors, the excited atoms have a random distribution in the orientation angle $\varphi$, we must take this into account.

If $t$ is the tunnel crossing time, the fraction $f(\theta)$ of atoms which survive after passing through the gap between the mirrors will be

$$
f(\theta)=\frac{1}{2} \int_{0}^{\pi} d \varphi \sin \varphi \exp \left[-t \Gamma_{\text {eff }}(\theta, \varphi)\right],
$$

where the integral over $\varphi$ means that we are averaging over the initial orientation of the atomic dipole. The fraction $f(\theta)$ can be compared with the experimental data, as we shall see.

Another way of expressing $f(\theta)$ is

$$
f(\theta)=\exp \left(-\left(\gamma_{\|}+\gamma_{\perp}\right) \frac{t}{2}-\left(\gamma_{\|}-\gamma_{\perp}\right) \frac{t}{4} \sin ^{2} \theta\right) I(\theta),
$$

and the function $I(\theta)$ is defined as

$$
I(\theta)=\int_{0}^{1} d u \exp \left(A u^{2}\right),
$$

where

$$
A=\frac{t}{2}\left(\gamma_{\|}-\gamma_{\perp}\right)\left(1-\frac{3}{2} \sin ^{2} \theta\right) .
$$

If $A<0$ the integral (3.8) corresponds to the wellknown error function. If, however, $A>0$, (3.8) corresponds to Dawson's integral [37]. In both cases it must be evaluated numerically.

The latter experiment above [36] is characterized by a value of $t$ (average tunnel crossing time) such that $t \gamma_{\mathrm{FS}}=12.8$ where, as before, $\gamma_{\mathrm{FS}}$ is the damping constant of the excited state $5 D_{1 / 2}$ in free space. In our calculation we consider $t \gamma_{\mathrm{FS}}$ as given since we are not able to obtain $\gamma_{\mathrm{FS}}$ for this state within the framework of SED [see (2.2)]. Since in this experiment $\lambda=3.49 \mu \mathrm{m}$ and the mirrors separation is $a=1.1 \mu \mathrm{m}$, we have $\omega a / \pi c=2 a / \lambda=0.63$. Therefore, according to the previous results (2.3) and (2.4), we get $\gamma_{\|}=0$ and $\gamma_{\perp} / \gamma_{\mathrm{FS}}=3 \lambda / 4 a=2.38$.

The comparison between our model calculation which leads to (3.7) and the experimental data is shown in Fig. 2. The agreement between our classical calculation based on SED and the experimental observations is very good. The corresponding QED calculation based on the LambBethe theory also presents a very good agreement with

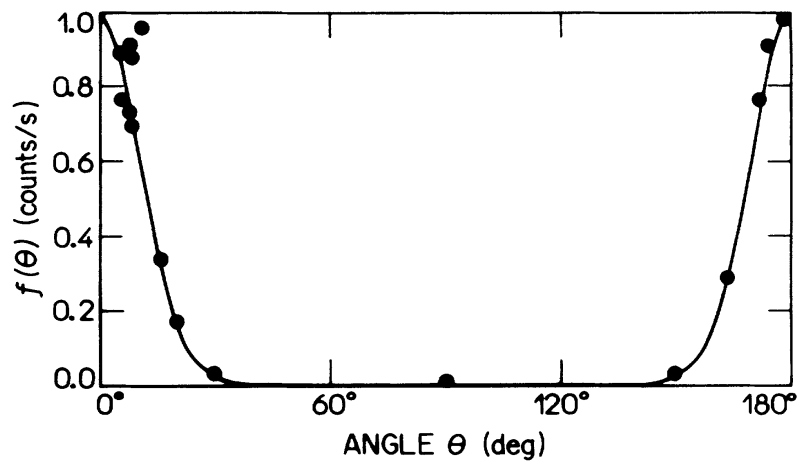

FIG. 2. Excited-state transmission between the mirrors as a function of the angle $\theta$ between the magnetic field and the normal to the plates. Solid line corresponds to (3.7) normalized to the maximum counting rate (30 counts per second [36]).

the experimental data reported in Ref. [36]. However, as far as we know, these quantum calculations have not been published $[9,36]$.

\section{DISCUSSION}

The phenomena discussed in the preceding section are very stimulating. One can conclude that physicists are learning to control the atomic behavior. If one has an atom (or molecule) between mirrors or inside resonant cavities [9], one can modify the behavior (in a probabilistic sense) of the microscopic system.

In the case of two parallel mirrors, for instance, we know that atomic excited states, which emit radiation (and also absorb energy from the zero-point electromagnetic fields) at a wavelength whose normal modes are affected by the cavity boundaries, have different properties from the same atomic state in free space. Not only is the lifetime affected. If the atom stays for a long time inside the cavity some states are "eliminated" because they become more unstable, while other states acquire the status of "stable states." From the simple example we have discussed one can infer that some states with large- $z$ components of the angular momentum become more stable because the electron trajectory is parallel to the plates and have inhibited emission of radiation $\left(\gamma_{\|}=0\right)$. This is interesting because one can modify the chemical properties of atoms and molecules by putting them inside appropriate cavities. The technological implications of this kind of "cavity chemical dynamics" is obviously attractive.

However, we want to draw the reader's attention to other fundamental implications. We have just seen that an unstable excited state can become stable (that is, one can increase its lifetime by a factor of 20 as has been observed in some experiments [35]) if we put the atom inside an appropriate environment. We understand this in terms of a balance between the emitted radiation and the energy absorbed from the environmental radiation, that is, the zero-point electromagnetic fields which corresponds to the allowed normal modes associated to the environment. This may be considered a typical classical (stochastic) mechanism for atomic stability. Therefore 
the natural questions which appear immediately are the following: What occurs with an atom in free space? Is it possible to understand the stability of the atomic ground state in the same manner?

In our opinion the answer to these questions may be simple, as we have already said in the Introduction. It is obvious that isolated systems do not exist. Therefore the environmental radiation in free space is simply the emitted radiation which comes from distant matter in the universe. Such a proposal, as an explanation for the observed zero-point electromagnetic fields, has been discussed more than once in the past $[38,39]$. As far as the second question is concerned, we also believe that the answer may be affirmative [20,40-42].

In this article we have tried to show how two ideas originating within the entirely classical concepts of stochastic electrodynamics have paved the way towards a deeper understanding of the interaction between atoms and the radiation field. The rate of such processes may be calculated with extraordinary accuracy in quantum electrodynamics, but their explanation remains obscure.

The first of these ideas is the understanding of the stationary states of nonrelativistic quantum mechanics, and the second is the related question of the status of the canonical commutation relations between position and momentum. Both of these have to be understood in a dynamical sense; the commutation relations can be maintained for all times only by introducing both the radiation reaction field and the zero-point field. This was recognized first within quantum electrodynamics by the group of Ackerhalt, Knight, and Eberly [43] and Milonni [12], but had been well recognized previously by the school of stochastic electrodynamics. The more recent recognization, by Dalibard, Dupont-Roc, and CohenTannoudji [13] and others [14], that a full understanding is possible only by working with the symmetrically ordered product of field and atom operators (leading to a full recognition that the radiation reaction and the zeropoint fields have a separate real existence) has led to an even closer convergence between QED and SED. One can now recognize not only why the ground state is the only truly stationary state, but also that the stability of the ground state itself results from the existence of the zero-point field.

We remark here that the fashionable area of research now known as cavity quantum electrodynamics was also foreseen in stochastic electrodynamics, since Marshall showed that, if we assume that a harmonic oscillator keeps the same ground state in a cavity as it has in free space, then this necessarily means replacing the zeropoint field of free space by one in which each cavity mode of oscillation is occupied by radiation of average intensity equivalent to half a "photon." We also remark that, in a subsequent article, Henry and Marshall [44] extended this idea to calculate the zero-point field in a cavity of finite quality factor $Q$, something that, so far as we know, has not been done in quantum electrodynamics. However, it is already seen from the article of Henry and Marshall that the statement [36], made frequently in cavity electrodynamics, that modes with wavelength greater than the dimension of the cavity $(\lambda>2 a$ in our example) are completely extinguished, has to be modified. In a cavity of finite $Q$ there is always some residual noise at all frequencies. But, by the fluctuation-dissipation theorem, the corresponding relaxation time for an oscillator with such frequencies will be very much greater inside the cavity than in free space.

We have to recognize, however, that, perhaps because its objective is to understand, (rather than merely calculate) physical phenomena, stochastic electrodynamics has not made any advances in the calculation of atomic energy levels. Indeed all the evidence is that the simple Braffort-Marshall equation of stochastic electrodynamics is not adequate for the electron in a Coulomb field $[41,42]$. Nevertheless, we can claim that there is some virtue in restricting attention to models based on the system which stochastic electrodynamics understands really well - the harmonic oscillator. In this context the article by Cray, Shih, and Milonni [27], together with references quoted therein, is very illuminating. It is essentially in accordance with such ideas that we have introduced our simple model with circular orbits in Sec. III.

The proposition that the intuitive insights of stochastic electrodynamics have something to offer, even in helping to understand such a monumental computational achievement as the QED calculation of gyromagnetic factor for the electron $(g-2)$, is well illustrated by CohenTannoudji [14]. There it is shown that the Lamb shift is mainly due to vacuum fluctuations, but the spin anomaly $g-2$ is mainly due to radiation reaction. In other words, the leading term of $g-2$ is given, in both sign and magnitude, by an interaction of the spin with the vacuum fluctuations and the radiation reaction field, that is, both are necessary.

\section{ACKNOWLEDGMENTS}

This work was partially supported by DGICYT (Spain) Grant No. PB 87-0014. One of us (H.M.F.) acknowledges the hospitality of the Universidad de Cantabria and also the financial support from Fundação de Amparo a Pesquisa do Estado de São Paulo (FAPESP), Conselho Nacional de Desenvolvimento Cientifico e Tecnológico (CNPq), and programa Banco Interamericano de Desenvolvimento - Universidade de Săo Paulo.
*Permanent address: Instituto de Física, Universidade de São Paulo, São Paulo, Brazil.

†Permanent address: Department of Mathematics, University of Manchester, Manchester, England.

[1] M. Planck, Ver. Deutsch. Phys. Ges. 13, 138 (1911); see P. W. Milonni and M. L. Shih, Am. J. Phys. 59, 684 (1991), for interesting comments concerning Planck's papers and the appearance of the zero-point energy in quantum theory.

[2] W. Nernst, Ver. Deutsch. Phys. Ges. 18, 83 (1916). In this remarkable paper, which is an "attempt to return to continuous changes in energy," Nernst discusses the role of the zero-point electromagnetic radiation in many different phenomena such as, for instance, atomic stability, specific 
heat of solids and degenerate gases, electron Zitterbewegung, Casimir and van der Waals forces, etc.

[3] Selected Papers on Quantum Electrodynamics, edited by J. Schwinger (Dover, New York, 1958).

[4] H. B. Casimir and D. Polder, Phys. Rev. 73, 360 (1948).

[5] T. A. Welton, Phys. Rev. 74, 1157 (1948).

[6] A. A. Sokolov and V. S. Tumanov, Zh. Eksp. Teor. Fiz. 30, 802 (1956) [Sov. Phys. —JETP 3, 958 (1956)].

[7] W. E. Lamb, Jr. and R. C. Retherford, Phys. Rev. 72, 241 (1947).

[8] M. J. Sparnaay, Physica 24, 751 (1958).

[9] S. Haroche and D. Kleppner, Phys. Today 42 (1), 24 (1989).

[10] R. Schiller and H. Tesser, Phys. Rev. A 3, 2035 (1971).

[11] W. Eckardt, Z. Phys. B 64, 515 (1986).

[12] P. W. Milonni, Phys. Scr. T21, 102 (1988); Am. J. Phys. 52, 340 (1984).

[13] J. Dalibard, J. Dupont-Roc, and C. Cohen-Tannoudji; J. Phys. (Paris) 43, 1617 (1982).

[14] Claude Cohen-Tannoudji, Phys. Scr. T12, 19 (1986).

[15] T. W. Marshall, Proc. R. Soc. London Ser. A 273, 475 (1963).

[16] T. W. Marshall, Nuovo Cimento 38, 206 (1965).

[17] E. Santos, Nuovo Cimento B 19, 57 (1974).

[18] T. H. Boyer, Phys. Rev. D 11, 790 (1975); 11, 809 (1975).

[19] P. W. Milonni, Phys. Rep. 25, 1 (1976).

[20] P. Claverie and S. Diner, Int. J. Quantum Chemistry Suppl. 12, 41 (1977).

[21] T. H. Boyer, in Foundations in Radiation Theory and Quantum Electrodynamics, edited by A. O. Barut (Plenum, New York, 1980), p. 49; see also Sci. Am. 253, 56 (1985).

[22] L. de la Peña, in Stochastic Processes Applied to Physics and Other Related Fields, edited by B. Gómez et al. (World Scientific, Singapore, 1982), p. 428.

[23] E. Santos, in Problems in Quantum Physics II; Gdańsk '89, edited by J. Mizerski, A. Posiewnik, J. Pykacz, and M. Zukowski (World Scientific, Singapore, 1990), p. 131; see also J. J. Sakurai Advanced Quantum Mechanics (Addison-Wesley, Reading, MA, 1967), p. 67.

[24] See T. H. Boyer, Phys. Rev. A 29, 2389 (1984); A. V. Barranco, S. A. Brunini, and H. M. França, ibid. 39, 5492 (1989).
[25] R. Blanco, H. M. França, and E. Santos, Phys. Rev. A 42, 693 (1991).

[26] See, T. W. Marshall and E. Santos, Phys. Rev. A 39, 6271 (1989), for a local realistic analysis of optical tests of Bell inequalities. See also A. V. Barranco and H. M. França, Found. Phys. Lett. 5, 25 (1992) and Phys. Essays 3, 53 (1990), for discussion of the Compton effect within the realm of classical stochastic electrodynamics.

[27] M. Cray, M. L. Shih, and P. W. Milonni, Am. J. Phys. 50, 1016 (1982).

[28] P. W. Milonni and P. L. Knight, Opt. Commun. 9, 119 (1973).

[29] A. M. Cetto and L. de la Peña, Phys. Rev. A 37, 1952 (1988); 37, 1960 (1988).

[30] J. D. Jackson, Classical Electrodynamics (Wiley, New York, 1975), Chap. 9.

[31] See J. D. Jackson, Classical Electrodynamics (Ref. [30]), Chap. 17.

[32] T. H. Boyer, Phys. Rev. 180, 19 (1969).

[33] M. H. França and T. W. Marshall, Phys. Rev. A 38, 6272 (1988).

[34] H. M. França and M. T. Thomaz, Phys. Rev. D 31, 1337 (1985); 38, 2651 (1988).

[35] R. G. Hulet, E. S. Hilfer, and D. Kleppner, Phys. Rev. Lett. 55, 2137 (1985).

[36] W. Jhe, A. Anderson, E. A. Hinds, D. Mesched, L. Moi, and S. Haroche, Phys. Rev. Lett. 58, 666 (1987).

[37] Handbook of Mathematical Functions, edited by $\mathbf{M}$. Abramowitz and Irene A. Stegun (Dover, New York, 1970), Chap. 7.

[38] E. Santos, An. Fis. (Madrid) 9, 317 (1968); E. Santos in Open questions in Quantum Physics, edited by G. Tarozzi and A. van der Merwe (Reidel, Dordrecht, 1985), p. 283.

[39] H. E. Puthoff, Phys. Rev. A 40, 4857 (1989); see also the Comment by E. Santos, Phys. Rev. A 44, 3383 (1991).

[40] H. E. Puthoff, Phys. Rev. D 35, 3266 (1987).

[41] T. W. Marshall and P. Claverie, J. Math. Phys. 21, 1819 (1980).

[42] P. Claverie and F. Soto, J. Math. Phys. 23, 753 (1982).

[43] J. R. Ackerhalt, P. L. Knight, and J. H. Eberly, Phys. Rev. Lett. 30, 456 (1973).

[44] L. L. Henry and T. W. Marshall, Nuovo Cimento 41, 188 (1966). 